\title{
Activity-Dependent Depression of Excitability and Calcium Transients in the Neurohypophysis Suggests a Model of "Stuttering Conduction"
}

\author{
Martin Muschol, ${ }^{1}$ Paul Kosterin, ${ }^{1}$ Michinori Ichikawa, ${ }^{3}$ and B. M. Salzberg ${ }^{1,2}$ \\ Departments of ${ }^{1}$ Neuroscience and ${ }^{2}$ Physiology, University of Pennsylvania, Philadelphia, Pennsylvania 19104-6074, and ${ }^{3}$ RIKEN Brain Research Institute, \\ Saitama 351-0198, Japan
}

\begin{abstract}
Using millisecond time-resolved optical recordings of transmembrane voltage and intraterminal calcium, we have determined how activity-dependent changes in the population action potential are related to a concurrent modulation of calcium transients in the neurohypophysis. We find that repetitive stimulation dramatically alters the amplitude of the population action potential and significantly increases its temporal dispersion. The population action potentials and the calcium transients exhibit well correlated frequencydependent amplitude depression, with broadening of the action potential playing only a limited role. High-speed camera recordings indicate that the magnitude of the spike modulation is uniform throughout the neurohypophysis, thereby excluding propagation failure as the underlying mechanism. In contrast, temporal dispersion and latency of the population spike do increase with distance from the stimulation site. This increase is enhanced during repeated stimulation and by raising the stimulation frequency. Changes in Ca influx directly affect the decline in population spike amplitude, consistent with electrophysiological measurements of the local loss of excitability in nerve terminals and varicosities, mediated by a Ca-activated $\mathrm{K}$ conductance. Our observations suggest a model of "stuttering conduction": repeated action potential stimulation causes excitability failures limited to nerve terminals and varicosities, which account for the rapid decline in the population spike amplitude. These failures, however, do not block action potential propagation but generate the cumulative increases in spike latency.
\end{abstract}

Key words: neurohypophysis; excitation-secretion coupling; action potential; calcium indicator; voltage-sensitive dye; temporal dispersion

\section{Introduction}

The peptide hormones arginine vasopressin and oxytocin are released from separate nerve terminals of the neurohypophysis, with optimal secretion of each determined by a different pattern of action potential stimulation (Poulain and Wakerley, 1982; Cazalis et al., 1985; Gainer et al., 1986; Bicknell, 1988). A variety of mechanisms have been proposed to account for this frequency dependence of release, including action potential broadening (Gainer et al., 1986, Bourque, 1990; Jackson et al., 1991), loss of excitability in nerve terminals and swellings (Bourque, 1990; Bielefeldt and Jackson, 1993), Ca channel inactivation (Branchaw et al., 1997), calcium buffering and extrusion (Stuenkel, 1994), and their impact on residual calcium accumulation (Jackson et al., 1991). It has been difficult to decide, however, to what extent any of the proposed mechanisms contribute to the frequency dependence of release measured from the intact tissue.

Received May 23, 2003; revised Sept. 26, 2003; accepted Sept. 27, 2003.

This work was supported by National Institutes of Health (NIH) Grants NS40966 and NS16824 and NIH Career Development Award NS02176 to M.M. We thank Dr. Phil Haydon and Dr. Mikey Nusbaum for critical comments and helpful suggestions on this manuscript.

Correspondence should be addressed to Dr. Brian M. Salzberg, Department of Neuroscience, University of Pennsylvania, 234 Stemmler Hall, 35th Street and Hamilton Walk, Philadelphia, PA 19104-6074. E-mail: bmsalzbe@mail.med.upenn.edu.

Copyright $\odot 2003$ Society for Neuroscience 0270-6474/03/2311352-11\$15.00/0
Using millisecond time-resolved optical recordings, we have recently determined detailed kinetics of tissue-averaged calcium elevations (Muschol and Salzberg, 2000). We found that the amplitude of the brief, stimulus-induced calcium transients displayed a pronounced pattern of facilitation and depression, varying with both the frequency and ordinality (position) of the individual stimuli within the train. The cellular basis for this pattern of calcium modulation, however, remained uncertain. In particular, the optical recordings represent the average response from a population of nerve terminals, secretory swellings, and axons. Therefore, the observed amplitude modulation could reflect either changes in the pattern of intraterminal calcium elevation (influx, release, buffering, and extrusion), changes in action potential waveform, or changes in the number of terminals activated by a given stimulus.

Here we have used voltage-sensitive dyes to measure transmembrane voltage changes during repetitive field stimulation. Optical recording using fast potentiometric dyes is a powerful technique for detecting global changes in action potential generation and propagation in an intact neurosecretory tissue and for assessing their effect on peptide release. In this study, we have used either a single PIN photodiode to monitor the voltage responses averaged over the whole neurohypophysis or a newly developed high-speed CMOS camera to determine the spatio- 
temporal pattern of action potential propagation and invasion. The goal of the present work was to address the following questions: What types of changes occur in the population action potential under physiological stimulation conditions, viz. trains of action potentials? How do these changes depend on frequency, and how does this frequency dependence compare with that of the calcium transients measured under the same stimulation conditions (Muschol and Salzberg, 2000)? Finally, can local failure of excitability in nerve swellings and terminals (Bourque, 1990; Bielefeldt and Jackson, 1993) be related to globally observed changes in the population action potential and to spike propagation and invasion throughout the axonal arbor?

\section{Materials and Methods}

Preparation and apparatus. Details of the preparation and apparatus have been reported previously (Salzberg et al., 1983; Gainer et al., 1986; Muschol and Salzberg, 2000). Typically, 30- to 60-d-old CD-1 female mice (The Jackson Laboratory, Bar Harbor, ME) were killed using $\mathrm{CO}_{2}$ asphyxiation followed by decapitation. The pituitary gland was removed, and the intact neurohypophysis, together with part of the infundibular stalk and the pars intermedia, were separated from the anterior pituitary. The neurohypophysis was mounted in an optical recording chamber and superfused with physiological saline containing (in mM): $155 \mathrm{NaCl}, 5.6$ $\mathrm{KCl}, 2.2 \mathrm{CaCl}_{2}, 1 \mathrm{MgCl}_{2}, 10$ glucose, and 20 HEPES, pH adjusted to 7.4, at a rate of $200 \mu \mathrm{l} / \mathrm{min}$. Axons, nerve terminals, and in-line varicosities are approximately uniformly distributed throughout the neurohypophysis. The preparation was excited by direct-field stimulation of the axons just as they branch into this heterogeneous tissue. Trains of stimuli were created with a Master- 8 pulse generator (AMPI Jerusalem, Israel) driving two ISO-Flex stimulus isolators (AMPI). Two platinum-iridium (90 and $10 \%$ ) electrodes delivered these balanced bipolar stimuli of $1 \mathrm{msec}$ total duration to the tissue. The voltage drop across the preparation was approximately $\pm 10 \mathrm{~V}$ and remained constant throughout the train. This stimulation protocol avoided electrode polarization artifacts and solution electrolysis. All experiments were performed at room temperature $\left(22 \pm 2^{\circ} \mathrm{C}\right)$, and all chemicals used were from Sigma (St. Louis. MO) unless otherwise indicated.

Optical measurements of changes in cytosolic calcium and transmembrane voltage. All fluorescence measurements were performed using a UEM upright microscope (Zeiss, Oberkochen, Germany) using a $20 \times$, 0.5 numerical aperture, water immersion objective (Leica, Bensheim, Germany) and a $300 \mathrm{~W}$ tungsten-halogen lamp as the epi-illumination source. For calcium measurements, the preparation was incubated for 90 min in a Ringer's solution containing $10 \mu \mathrm{M}$ of the low-affinity $\left(K_{\mathrm{D}, \mathrm{Ca}}, 22\right.$ $\mu \mathrm{M}$ ) calcium indicator dye mag-fluo-4 AM (Molecular Probes, Eugene, OR), $0.9 \%$ dimethylsulfoxide, and $0.1 \%$ Pluronic F-127 (BASF, Wyandotte, MI). The filter set consisted of a $470 \pm 20 \mathrm{~nm}$ excitation filter, a 500 $\mathrm{nm}$ dichroic mirror (Chroma Technology, Brattleboro, VT), and a 515 nm long-pass filter (Schott Optical Glass Co., Duryea, PA). For optical measurement of transmembrane voltage, the neurohypophysis was incubated for 30-40 min in a Ringer's solution containing $20 \mu \mathrm{M}$ of the fast-response potentiometric styryl dye JPW 1114 (Molecular Probes). The filter set used for JPW 1114 was a $530 \pm 45 \mathrm{~nm}$ excitation filter (Corion, Holliston, MA), a $585 \mathrm{~nm}$ dichroic mirror (Chroma Technology), and a $610 \mathrm{~nm}$ long-pass filter (Schott Optical Glass Co.). For anterograde loading of JPW 1114, the dye was dissolved at $5 \mathrm{mg} / \mathrm{ml}$ in an intracellular medium containing (in mM): $154 \mathrm{KCl}, 40$ HEPES-Tris, and 0.25 EGTA, pH 7.4. The preparation was placed in a loading chamber, and a short segment of the freshly cut end of the infundibular stalk was aspirated into a suction electrode filled with intracellular medium, which was then replaced with the concentrated dye stock. The preparation was incubated overnight in oxygenated $\left(95 \% \mathrm{O}_{2}\right.$ and $\left.5 \% \mathrm{CO}_{2}\right)$ cell medium (medium 199; Invitrogen, Rockville, MD).

Photodiode measurements. Tissue-averaged measurements of optical signals were obtained with a large-area photodiode (PV-444; PerkinElmer Optoelectronics, Vaudreuil, Quebec, Canada). The photocurrent was converted into a voltage signal with either a commercial current-to-voltage converter (DLPCA 200; Femto Messtechnik, Berlin
Germany) or a custom-built track-and-hold amplifier (Cellular and Molecular Physiology Electronics Shop, Yale University School of Medicine, New Haven, CT). The resulting voltage traces were low-pass filtered with an eight-pole Bessel filter (440; Brownlee Precision, Santa Clara, CA) and digitized at 16-bit resolution using a data acquisition board (AT-MIO16XE-50; National Instruments, Austin, TX). Typical low-pass filter/data sampling pairs were $500 \mathrm{~Hz}$ and $1 \mathrm{kHz}$ for calcium changes and 1 and 2 $\mathrm{kHz}$ for voltage recordings.

High-speed camera recordings. Spatially resolved multiple site optical recordings of transmembrane voltage were obtained using a high-speed (up to $10 \mathrm{kHz}$ frame rate) $100 \times 100$ pixel CMOS image sensor and a fast image acquisition system (developed by RIKEN Brain Science Institute and Stanley Electric Co. Ltd.) interfaced with a personal computer using proprietary camera software. The CMOS active-pixel image sensor uses high-fill factor technology to achieve both large well depth and good sensitivity; an individual pixel has a capacity of $>10$ million electrons, with a quantum efficiency of $\sim 60 \%$ for visual light. The dark noise of the camera is $700 \mathrm{e}^{-}(1 \mathrm{kHz})$ in the current prototype version, but a value of $400 \mathrm{e}^{-}$is anticipated in the final version. To realize high-speed readout, the sensor is equipped with 16 parallel outputs. These signals are input to 16 fast precision analog-to-digital converters, and the digital signals are stored in 512 megabyte memories via a wide (256-bit) data bus. A detailed system description can be found at http://www.brain.riken.go.jp/ labs/bod/imaging1.htm. In this study, images were acquired at a $1 \mathrm{kHz}$ frame rate in differential mode, i.e., with an image of the initial resting fluorescence subtracted from all subsequent frames. Traces were stored on CD-R disks for off-line processing.

Data analysis. All data analysis was performed using IGOR data analysis software (Wavemetrics, Lake Oswego, OR). For single-photodiode recordings, traces were typically bleach-corrected by linear extrapolation of a $1 \mathrm{sec}$ baseline segment at the beginning of the recording. The amplitudes of the individual fast calcium and voltage transients $\left(\Delta \mathrm{Ca}_{\mathrm{tr}}\right.$ and $\left.\Delta \mathrm{V}_{\mathrm{tr}}\right)$ represent the difference between the signal level preceding a stimulus and its peak value. Both voltage and calcium transients rise sufficiently fast to permit us to neglect possible contributions from various slow components of the optical traces (e.g., bleaching, afterdepolarization, and residual calcium). The amplitudes of the transients were not significantly affected by changes in action potential waveform. For camera recordings, false-color images of the sequence of frames for each compound action potential were generated using IGOR. These sequences were then corrected for the slow accumulation of the spatial heterogeneities in dye fluorescence discussed in Corrections of camera data.

Consideration of dye properties. For a simple interpretation of the changes in the amplitude of the optical signals, the respective indicator dyes have to respond linearly to membrane voltage and to calcium concentration changes. We have previously confirmed that mag-fluo-4 $\left(K_{\mathrm{D}, \mathrm{Ca}}, 22 \mu \mathrm{M}\right)$ operated clearly inside its linear response range in the neurohypophysis (Muschol and Salzberg, 2000). In vitro measurements of the $\mathrm{Ca}^{2+}$ affinity of mag-fluo-4 exhibited no significant $\mathrm{pH}$ sensitivity (data not shown), in agreement with the behavior seen with other lowaffinity indicator dyes (Lattanzio and Bartschat, 1991). Further details concerning the interpretation of the optical signals from calcium indicator dyes have been discussed previously (Muschol and Salzberg, 2000). Potentiometric dyes are well established linear probes of membrane potential (Cohen and Salzberg, 1978; Salzberg, 1983). Using a variety of fast potentiometric styryl dyes (JPW 1114, RH 414, RH 795, RH 424), we did not observe any significant differences in the shape or time course of the optical compound action potential (other than the relative amplitude of the afterdepolarization; see Results). JPW 1114 was selected for its superior signal-to-noise ratio, its limited phototoxicity, and the comparatively small amplitude of the afterdepolarization.

Phototoxicity poses an experimental problem for all fluorescent dyes. Preparations stained internally with JPW 1114 were especially sensitive, with signal waveforms altered after one or two brief light exposures. All data from externally stained preparations were collected at light intensities that yielded stable and reproducible recordings for several hours. Unlike increases in light intensity, changes in dye concentration (2-25 $\mu \mathrm{M})$, solvent concentration (0.5-2\% DMSO), or incubation period (20- 
$120 \mathrm{~min}$ ) had no discernable effect on the shape or time course of the fluorescence signals in this preparation. As a further precaution, we collected a reference signal (typically $15 \mathrm{~Hz}$ stimulation 40 times) throughout the experiment. If the amplitude modulation of this reference signal changed, the data were rejected.

Immediately after staining the preparation with JPW 1114, the fluorescence background intensity exhibited an initial increase with time instead of the decrease expected from bleaching. Once dye loss had reduced the membrane-bound dye concentration sufficiently, normal bleaching was observed. This behavior is consistent with dye unquenching. At the initial concentration in the membrane, dye fluorescence is significantly quenched. Repartition of the dye into the bath gradually relieves this dye quenching, resulting in the observed net increase in fluorescence intensity and, eventually, in the return to normal bleaching behavior.

Corrections of camera data. As in the case of the single-photodiode data, several slow components of fluorescence change were superimposed on the camera recordings of fast voltage transients. These slow components generated systematic shifts in the false-color scale, distorting the visual impression of action potential invasion. Besides bleaching, unquenching, and the glial afterdepolarization (see Results), the camera data revealed an additional, activity-dependent change in the fluorescence background. The amplitude of this slow modulation varied across the preparation (e.g., see background modulation in Fig. 6A, top, center, bottom traces). This developing background heterogeneity is most likely the result of activity-dependent dye internalization (Rohr and Salzberg, 1994) by mechanisms that include membrane recycling similar to that observed with the dye FM 1-43 (Betz and Bewick, 1992). Here, again, we were able to exploit the large difference in time scales to separate the fast transients from any slow signal contamination. We used the camera frame just before a given stimulus as an "instantaneous" reference, subtracting it from the subsequent short sequence of frames containing the stimulus-induced voltage transient, thereby removing any accumulated slow modulation.

\section{Results}

\section{Optical recordings of voltage changes from} the neurohypophysis

Fast potentiometric dyes provide optical signals that are linearly proportional to changes in transmembrane voltage (Cohen and Salzberg, 1978). Figure $1 A$ shows a representative trace of the fluorescence change recorded from the intact mouse neurohypophysis in response to a single stimulus using the fluorescent voltage-sensitive dye JPW 1114 (Antic et al., 1992; Antic and Zecevic, 1995). This optical record represents the voltage response of the axonal membrane (axon segments, neurosecretory swellings, and terminals) together with any other cell structures that become depolarized. After the action potential, a prominent afterdepolarization develops, which peaks $\sim 50 \mathrm{msec}$ after the action potential. A comparable afterdepolarization in optical recordings of the membrane potential from the molecular layer of the skate cerebellum has been identified as the glial cell response to local potassium accumulation (Konnerth et al., 1987). Two lines of evidence support the same interpretation here. First, similar to the observations in skate (Konnerth et al., 1987), potentiometric dyes with different-length alkyl side chains tend to yield different relative amplitudes for the afterdepolarization and the action potential (data not shown). This suggests that the optical signal arises from two distinct types of membranes having differential affinities for different potentiometric dyes. Independently, we were able to stain the axonal membrane (axon segments, varicosities, and nerve terminals) selectively by means of anterograde loading of the dye through the cut axons of the infundibular stalk (Fig. $1 B$ ). Internal loading reverses the orientation of this membrane-impermeant dye molecule in the cell membrane, thereby changing the sign of the fast fluorescence signal (Davila et
A

B

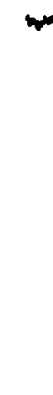
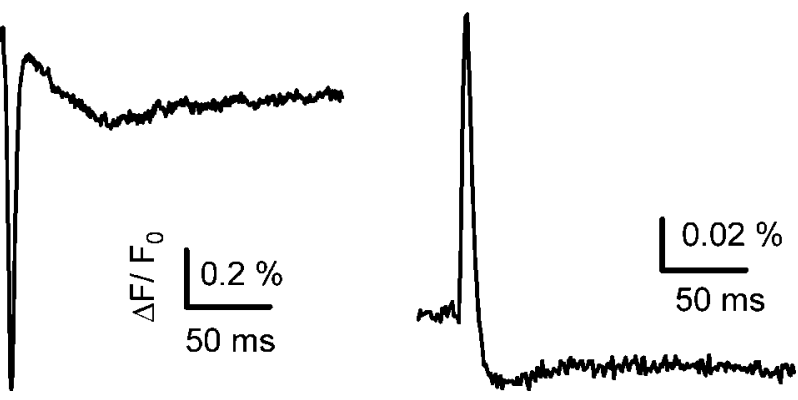

C

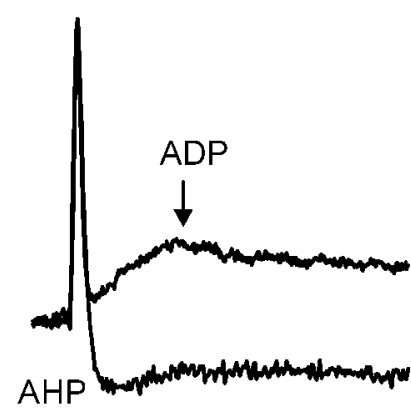

Figure 1. Optical recording of membrane potential in the neurohypophysis: external versus internal dye staining. $A$, Individual population action potential from the intact mouse neurohypophysis, recorded optically with a large-area photodiode after external staining with the fast potentiometric dye JPW 1114. The prominent afterdepolarization (ADP) peaks 50 msec after the action potential transient and decays within $1 \mathrm{sec}$. $B$, Same signal as in $A$ after selective staining of the nerve terminals and axons by anterograde loading of JPW 1114 through the cut axons of the infundibular stalk. Because JPW 1114 is membrane-impermeant, internal loading versus external staining lead to opposite orientations of the dye molecule in the cell membrane, thereby reversing the signal polarity. $C$, Superposition of $A$ and $B$. After internal staining, the prominent afterdepolarization of the optical signal is replaced by an afterhyperpolarization (AHP), demonstrating that the afterdepolarization arises from the voltage response of nonaxonal membranes, most likely pituicytes (see Results). For details of data acquisition and processing, see Materials and Methods.

al., 1974). Superimposing the external and internal signals (Fig. $1 C)$, it is readily apparent that selective staining of the axonal membrane replaces the afterdepolarization with an afterhyperpolarization. Because pituicytes are the only other cell type contributing a significant fraction of the membrane area in the neurohypophysis (Nordmann, 1977), we interpret the afterdepolarization as the voltage response of this glial cell type to extracellular potassium accumulation.

Although eliminating the contaminating glial signal represented by the afterdepolarization, anterograde staining substantially increased phototoxicity and typically reduced signal-tonoise ratios. To avoid these problems we used external dye staining for most subsequent experiments. Fortunately, both the slow rise time (dye-independent) and the small amplitude (dyedependent) of the pituicyte signal relative to the action potential limited the distortion of the action potential waveform to the late phase $(>10 \mathrm{msec}$ ) of repolarization (Fig. 1C). During trains of stimuli, saturation of the glial response (Konnerth et al., 1987) further decreases the contribution of the afterdepolarization. Thus, to quantify overall excitability changes, we have used the amplitude of the extrinsic transient optical changes, $\Delta \mathrm{V}_{\mathrm{tr}}$, as measured from the onset of the voltage signal to the peak of the action potential. This approach to the separation of the glial from the 

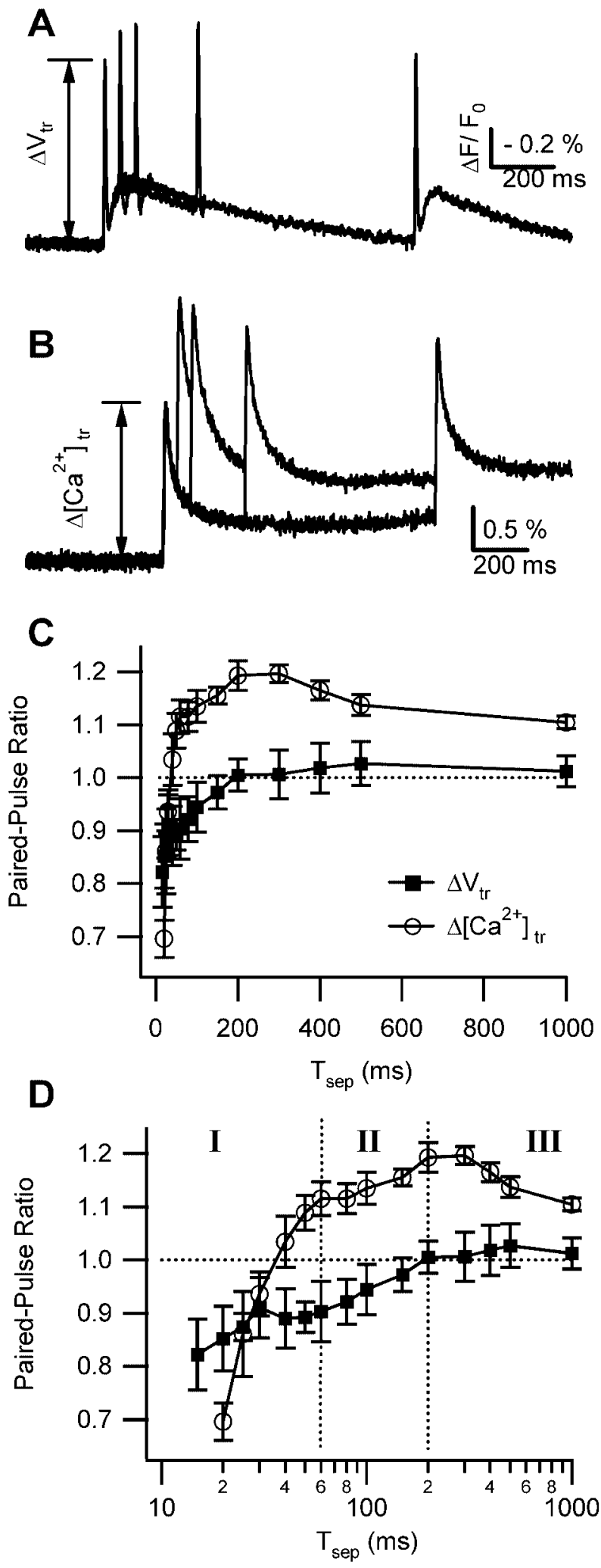

Figure 2. Paired pulse recordings of transmembrane voltage and calcium changes in the intact neurohypophysis. $A$, Spatially integrated voltage response of the neurohypophysis to a pair of stimuli at various pulse separations ( $T_{\text {sep }}=50,100,300 \mathrm{msec}$ and $1 \mathrm{sec}$ ). The peaks of the first transients, $\Delta \mathrm{V}_{\mathrm{tr}}$, in each pair are matched to one another and superimposed. Except for the $T=1$ sec trace, all records were truncated shortly after the second peak. The optical voltage signals have been inverted so that depolarization is shown as an upward deflection. Dye: JPW 1114, external staining. $B$, Calcium transients, $\Delta\left[\mathrm{Ca}^{2+}\right]_{\mathrm{tr}}$, in response to a pair of stimuli with the same pulse separations as in $A$. The fast-rising calcium transient, $\Delta\left[\mathrm{Ca}^{2+}\right]_{\mathrm{tr}}$, is neuronal signal is further validated by the observation that dye loss during optical measurement of membrane potential was selective, reducing the glial afterdepolarization much more rapidly than the fast optical signal from the neuronal membrane. This loss of glial signal, however, had no effect on the changes in $\Delta \mathrm{V}_{\mathrm{tr}}$ peak amplitudes detailed below.

\section{Voltage versus calcium transients during repeated stimulation}

One objective was to determine whether and how transmembrane voltage responses change during physiologically relevant episodes of repetitive firing. Also, if there are changes in the action potential, we wanted to explore how these changes might contribute to the observed frequency-dependent modulation of the associated calcium transients (Muschol and Salzberg, 2000). To this end, we measured and compared the paired pulse ratios for the tissue-averaged $\Delta \mathrm{V}$ and $\Delta\left[\mathrm{Ca}^{2+}\right]$ transients. We also compared the amplitude modulation of each of these signals as a function of stimulus frequency during prolonged trains of stimuli.

\section{Paired pulse modulation of $\Delta \mathrm{V}$ and $\Delta\left[\mathrm{Ca}^{2+}\right]$ transients}

Figure 2 displays representative optical recordings of membrane potential (Fig. $2 A$ ) and calcium transients (Fig. $2 B$ ) after pairs of stimuli at 4 of the 15 different pulse separations $\left(\mathrm{T}_{\text {sep }}\right)$ used for these measurements. The amplitudes of the fast transients of each optical trace were determined from the difference between the signal level immediately preceding the stimulus and its subsequent peak value. This procedure effectively eliminates the contribution from the slow component present in each of the optical signals (pituicyte afterdepolarization for $\Delta \mathrm{V}_{\text {tr }}$ and residual calcium rise and decay for $\Delta \mathrm{Ca}_{\mathrm{tr}}$ ) to the stimulus-induced amplitude changes.

Figure 2, $C$ and $D$, shows the paired pulse ratios of $\Delta \mathrm{V}_{\mathrm{tr}}$ and $\Delta\left[\mathrm{Ca}^{2+}\right]_{\text {tr }}$ versus pulse separation, $\mathrm{T}_{\text {sep }}$, on linear and logarithmic time scales. For clarity, we used a logarithmic time axis in Figure $2 \mathrm{D}$ and divided it into three separate time domains. $\Delta \mathrm{V}_{\text {tr }}$ paired pulse ratios facilitate only slightly $(<3 \%)$ in region III ( 200 msec $\left.\leq \mathrm{T}_{\text {sep }} \leq 1 \mathrm{sec}\right)$ but depress noticeably in regions II (60 msec $\left.\leq \mathrm{T}_{\text {sep }} \leq 200 \mathrm{msec}\right)$ and $\mathrm{I}\left(\mathrm{T}_{\text {sep }} \leq 60 \mathrm{msec}\right)$. In contrast, ratios of $\Delta\left[\mathrm{Ca}^{2+}\right]$ transients show prominent and persistent paired pulse facilitation in region III, peaking at $\sim 20 \%$. In region II, $\Delta \mathrm{Ca}_{\mathrm{tr}}$ and $\Delta \mathrm{V}_{\mathrm{tr}}$ ratios deteriorate at equal rates with decreasing pulse separation. For $\mathrm{T}_{\text {sep }}$ in region $\mathrm{I}$, the rate of $\Delta \mathrm{Ca}_{\mathrm{tr}}$ deterioration accelerates significantly as pulse separation decreases, eventually exceeding $\Delta \mathrm{V}_{\text {tr }}$ depression for $\mathrm{T}_{\text {sep }}$ of $<30 \mathrm{msec}$.

The separation of time scales for the onset of $\Delta \mathrm{Ca}_{\mathrm{tr}}$ facilitation versus depression implies that these two processes are activated by separate mechanisms. This conclusion is consistent with the observation that the recovery rates for $\Delta \mathrm{Ca}_{\mathrm{tr}}$ facilitation and depression during burst stimulation are equally distinct (Muschol and Salzberg, 2000). The absence of $\Delta \mathrm{V}_{\mathrm{tr}}$ facilitation in region III

$\leftarrow$

followed by a bimodal decay phase. The elevated baseline in the calcium signal originates from a very slow decay component not well resolved on this time scale (for details, see Muschol and Salzberg, 2000). All traces are peak-matched, superimposed, and truncated as in A. Dye: magfluo-4 AM. All records are low-pass filtered at $500 \mathrm{~Hz}$ and digitized at $1 \mathrm{kHz}$. C, Facilitation and depression of the paired pulse ratios of the $\Delta V$ (filled squares) and $\Delta\left[\mathrm{Ca}^{2+}\right]$ (open circles) transients as a function of the pulse separation, $T_{\text {sep }}$. The displayed ratios represent averages from several measurements $(\Delta \mathrm{V}, n=6 ; \Delta \mathrm{Ca}, n=3)$. Error bars indicate $1 \mathrm{SD}$ from the mean. For details of data processing, see Materials and Methods. D, Same data as in Con a logarithmic time scale. $\Delta \mathrm{V}_{\mathrm{tr}}$ and $\Delta\left[\mathrm{Ca}^{2+}\right]_{\mathrm{tr}}$ display different correlations in each of the three indicated time windows, I-III (see Results). 
A

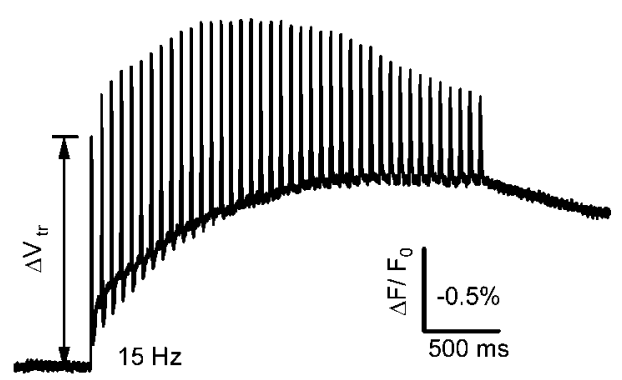

B

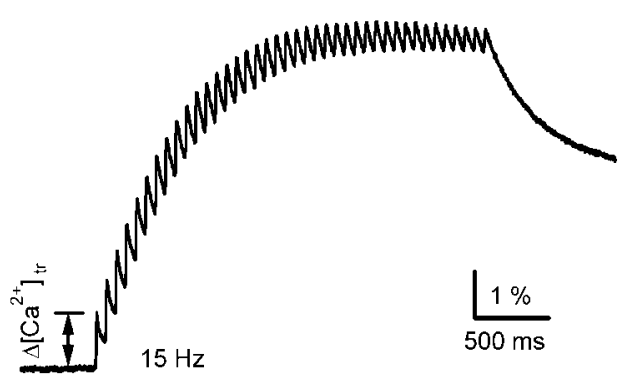

C
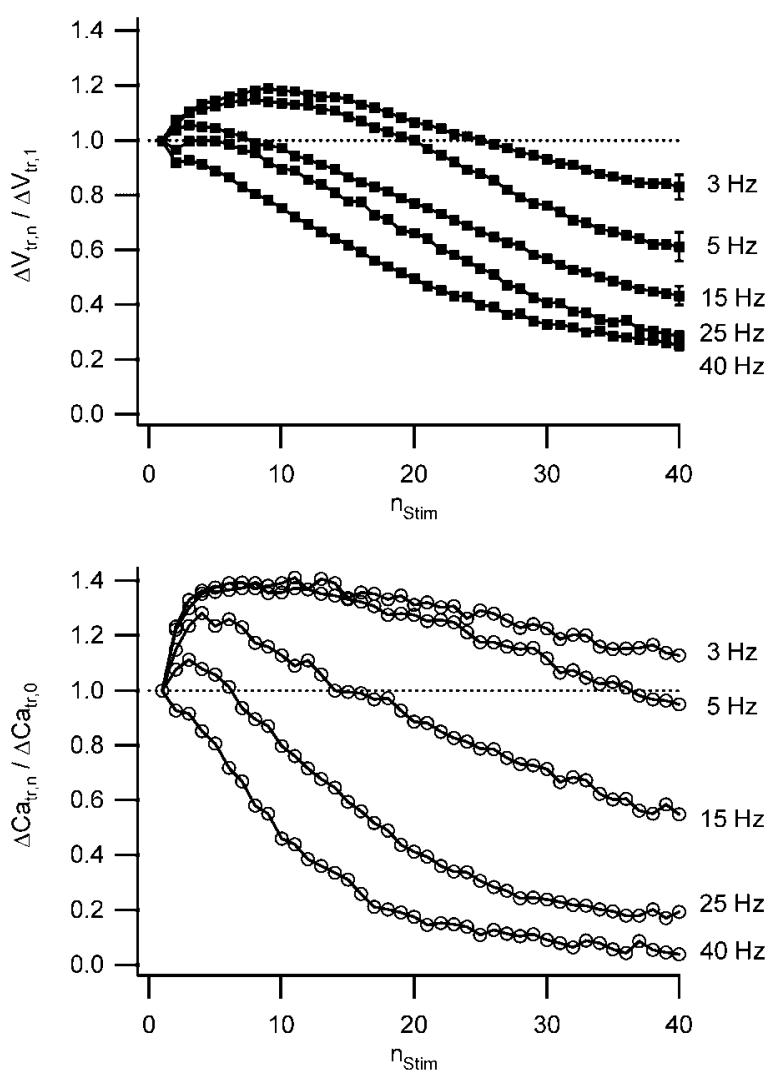

Figure 3. Frequency-dependent modulation of voltage and calcium transients during trains of action potentials. $A$, Representative trace of the voltage signal from the neurohypophysis in response to a train of 40 stimuli delivered at $15 \mathrm{~Hz}$. The rapid voltage transients rise from a slowly modulated increase in background fluorescence. The background increase consists of the glial afterdepolarization and contributions from activity-dependent dye internalization (e.g., membrane reuptake). $B$, Calcium changes recorded with the calcium indicator dye mag-fluo-4 AM under the same stimulation conditions as in A. C, Amplitude modulation of transmembrane voltage transients, relative to the first transient in a given train, at five different frequencies $(n=6)$. D, Modulation of the amplitude ratios of successive calcium transients, relative to the first transient in a given train (data from Muschol and Salzberg, 2000; error bars below symbol size). For details of measurements and data processing, see Materials and Methods. indicates that $\Delta \mathrm{Ca}_{\mathrm{tr}}$ facilitation is not directly related to changes in the population spike amplitude. The tight coupling of the onset and the rate of deterioration for $\Delta \mathrm{Ca}_{\mathrm{tr}}$ and $\Delta \mathrm{V}_{\mathrm{tr}}$ ratios in region II (60 msec $\left.\leq \mathrm{T}_{\text {sep }} \leq 200 \mathrm{msec}\right)$, in turn, suggest that $\Delta \mathrm{V}_{\mathrm{tr}}$ changes are responsible for the decline in $\Delta \mathrm{Ca}_{\mathrm{tr}}$ facilitation and the eventual onset of $\Delta \mathrm{Ca}_{\mathrm{tr}}$ depression in region $\mathrm{I}$.

The decline in the population spike amplitude with decreasing pulse separation, although surprisingly large, could indicate either a reduction in the peak amplitude of the action potential (partial refractoriness) or a loss of excitable membrane area resulting from changes in tissue excitability. Similarly, the correlation between $\Delta \mathrm{V}_{\mathrm{tr}}$ depression and the decline of $\Delta \mathrm{Ca}_{\mathrm{tr}}$ is suggestive but might not reflect a causal connection. We have shown previously (Muschol and Salzberg, 2000), however, that $\Delta \mathrm{Ca}_{\mathrm{tr}}$ amplitudes deteriorate by as much as $80 \%$ during trains of stimuli at $40 \mathrm{~Hz}$. A comparable decline in $\Delta \mathrm{V}_{\mathrm{tr}}$ amplitudes during repeated stimulation could no longer be reconciled with a decline in the amplitude of individual action potentials. Instead, it would strongly support the idea that the loss of excitable membrane area attributable to excitability and invasion changes dominates $\Delta \mathrm{V}_{\mathrm{tr}}$ (population) amplitude depression, and this decline contributes significantly to the depression of tissue-averaged calcium transients.

$\Delta \mathrm{V}_{\mathrm{tr}}$ and $\Delta \mathrm{Ca}_{\mathrm{tr}}$ modulation during trains of action potentials A characteristic voltage signal from the neurohypophysis in response to a train of 40 stimuli at $15 \mathrm{~Hz}$ is shown in Figure $3 \mathrm{~A}$. Each stimulus evokes a population action potential superimposed on a slowly increasing fluorescence background. The transients related to the action potential, $\Delta \mathrm{V}_{\mathrm{tr}}$, rise rapidly from this background and can be readily separated from it (Fig. $3 A$ ). The background increase consists of at least two components: the glial afterdepolarization, dominant at low frequencies, and activitydependent dye internalization (Rohr and Salzberg, 1994), prominent at higher frequencies (also see Materials and Methods). Measurements of calcium transients, $\Delta \mathrm{Ca}_{\mathrm{tr}}$, corresponding to the same stimulation conditions as in Figure $3 A$ are displayed in Figure $3 B$. Again, the rapid rising phase of $\Delta \mathrm{Ca}_{\mathrm{tr}}$ is well separated from the slow increase in residual calcium.

The modulation of $\Delta \mathrm{V}_{\mathrm{tr}}$ and $\Delta \mathrm{Ca}_{\mathrm{tr}}$ amplitudes with ordinality, and at various stimulation frequencies, is summarized in Figure 3, $C$ and $D$, respectively. At the lowest stimulation frequencies $(\leq 5 \mathrm{~Hz})$, there is a modest facilitation of $\Delta \mathrm{V}_{\mathrm{tr}}$ during the first 20 stimuli. Beginning at $15 \mathrm{~Hz}$, the dominant effect is the pronounced depression of the population spike. The depression deepens throughout the stimulus train and reaches values as high as $75 \%$ after 40 stimuli at $40 \mathrm{~Hz}$. The dramatic decline in the population spike amplitude implies that a loss of excitable membrane area, and not a reduction in the peak amplitude of individual action potentials, dominates the observed $\Delta \mathrm{V}_{\mathrm{tr}}$ modulation. The modulation of $\Delta \mathrm{Ca}_{\mathrm{tr}}$ is identical to that reported previously (Muschol and Salzberg, 2000). Comparing the variation of the two signals, several features are evident. The facilitation of $\Delta \mathrm{V}_{\mathrm{tr}}$ at low frequencies is likely to contribute to but cannot fully explain the magnitude and persistence of the pronounced facilitation of $\Delta \mathrm{Ca}_{\mathrm{tr}}$ (past $n_{\text {stim }}=20$ ) in that frequency range. In contrast, the prominent depression of $\Delta \mathrm{V}_{\mathrm{tr}}$ correlates very well with the corresponding depression of $\Delta \mathrm{Ca}_{\mathrm{tr}}$ at frequencies of $>10 \mathrm{~Hz}$ (at $n_{\text {stim }}$ $\geq 20$ ). Similar to the paired pulse data, the rate of depression with ordinality of $\Delta \mathrm{Ca}_{\mathrm{tr}}$ at high frequencies exceeds the depression of $\Delta \mathrm{V}_{\mathrm{tr}}$, particularly early in the train. Nevertheless, the loss of excitability at moderate to high frequencies and during prolonged 

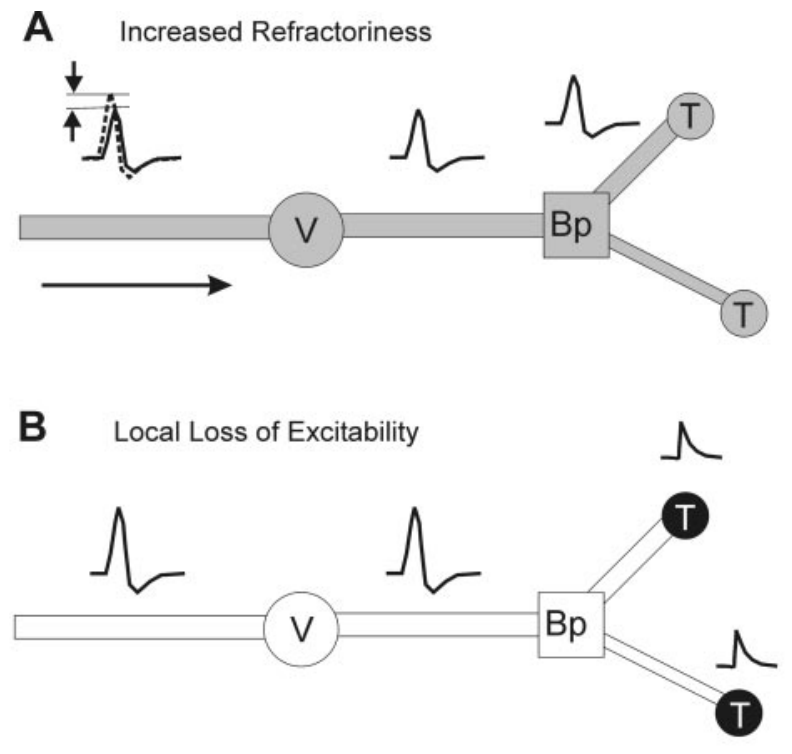

C Partial Failure of Spike Initiation
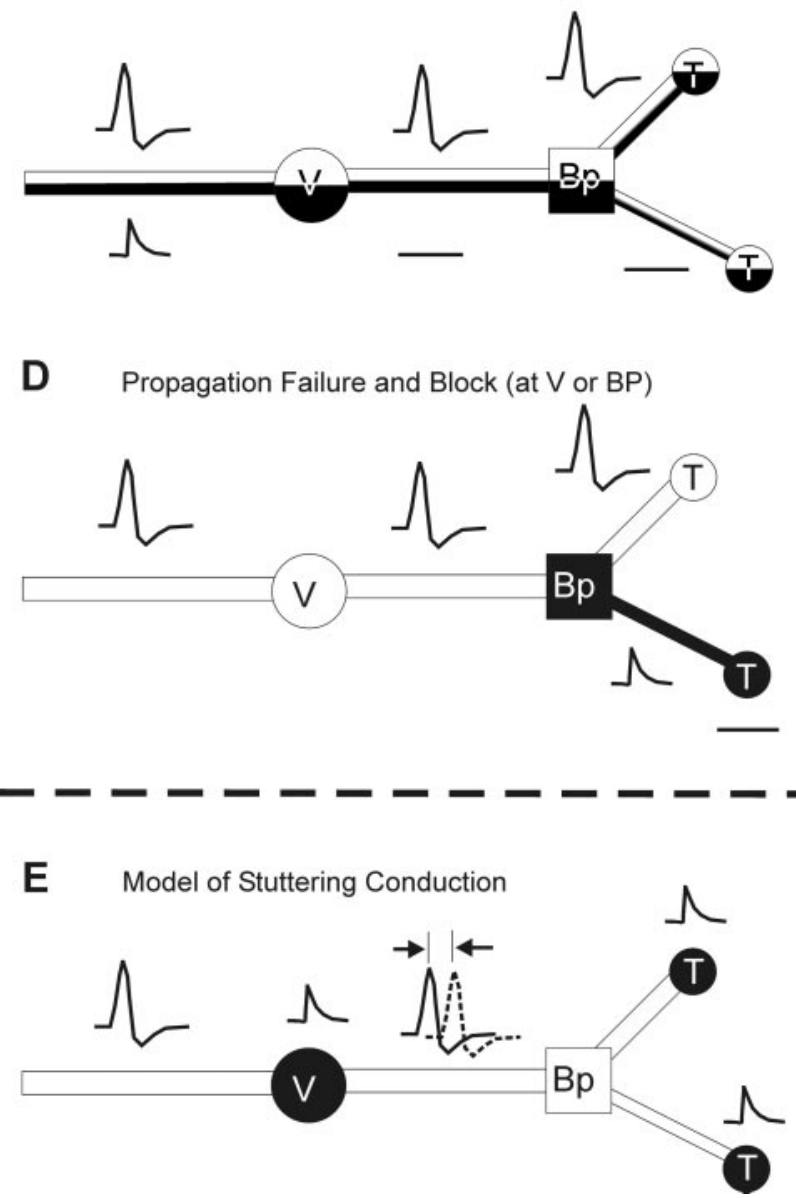

Figure 4. Models for the amplitude modulation of the population action potential: diagram representing the essential morphological features of the neurohypophysial axonal arbor (for actual axonal morphology displays, see Nordmann, 1977; Bourque, 1990; Jackson, 1993). Straight segments, Axon; V, in-line secretory varicosities; Bp, axonal branch points; T, nerve terminals. The waveforms above the axon indicate the local response (spike, active propagation; exponential or line, passive spread and decay) to a stimulus at the left margin. Shading indicates the spatial spread of inactivation: white, fully active; black, completely passive. $A$ Increased refractoriness. The sodium channels in the axons do not completely recover in between stimuli, thereby reducing the amplitude of the action potential. $B$, Local loss of excitability. Only nerve terminals become inexcitable (e.g., through the activation of a localized Ca-dependent stimulus trains can readily account for a significant fraction of the depression of calcium transients.

\section{Spatial properties of excitability changes}

Although the single-photodiode recordings establish the importance of $\Delta \mathrm{V}_{\mathrm{tr}}$ modulation, these data do not ascribe responsibility to any specific mechanism. Indeed, a variety of distinct mechanisms could account for the observed changes in the population action potential. On the basis of the actual axonal morphology (Bourque, 1990, his Fig. 10; Jackson, 1993, his Fig. 4), we have considered four different models, represented schematically in Figure 4. Axons (including their varicosities and terminals) might become partially refractory during repetitive stimulation, leading to a uniform decrease in action potential amplitude (Fig. 4, model $A$ ). Alternatively, the action potential may invade the whole axonal arbor (including varicosities) but may fail to evoke active responses locally in nerve terminals (Fig. 4, model B). An overall increase in the action potential threshold, with whole axons becoming refractory to spike initiation at the site of stimulation, provides yet another possible explanation (Fig. 4, model $C$ ). Finally, action potential propagation might be blocked in distal parts of the ramified axonal arbor (Obaid and Salzberg, 1996), with the rate of propagation failures depending on stimulation frequency (Fig. 4, model $D$ ).

Ideally, to distinguish between these different models, one should image action potential propagation in individual axonal branches. Because fast potentiometric dye signals are small in amplitude, and only dye molecules associated with the cell surface contribute to the signal, multiple-site optical recording of transmembrane voltage, in an intact tissue, with subcellular resolution (Rohr and Salzberg, 1994) is technically feasible only under special circumstances [e.g., intracellular injection of single somata (Antic et al., 1999) or extracellular staining of twodimensional networks (Obaid et al., 1999)]. These models do, however, predict distinct spatial patterns of action potential modulation that can be tested on a macroscopic scale. Because varicosities and terminals are distributed approximately uniformly along the axonal arbors in the neurohypophysis, and because even individual camera pixels record the average response from many of these structures, increased refractoriness (Fig. 4, model $A$ ), failures of spike initiation (Fig. 4, model $C$ ), or local loss of excitability at axonal terminals (Fig. 4, model $B$ ), should all result in a spatially uniformly reduction of the population spike amplitude. In Figure 4, model $D$, the number of in-line varicosities and branch points encountered by the action potential in-

\footnotetext{
$\leftarrow$

K-conductance; see Results). The action potential invades the nerve terminals passively. C, Partial failure of spike initiation. A fraction of the axonal branches becomes completely refractory to spike initiation at the site of stimulation, whereas others remain active (indicated by a split between black, white). D, Propagation failure and block. The action potential is blocked from invading the distal parts of the axon, by failing either at passivated varicosities or at branch points. Note that the axonal segments, nerve terminals, and varicosities are distributed approximately uniformly throughout the intact neurohypophysis. Therefore, models $A-C$ predict a uniform decline of locally averaged recordings of the transmembrane voltage, whereas model $D$ predicts increasing failures with distance from the stimulation site. $E$, Model of stuttering conduction (see Discussion). Varicosities and nerve terminals lose their excitability after repeated stimulation. The action potential invades these inactive structures passively but carries enough current to elicit an active response downstream. Each time the action potential passes through a passive varicosity, it picks up a small but finite delay; i.e., it stutters. Stuttering conduction readily accounts for both the spatially uniform decline in the amplitude of the locally averaged population action potential and the increase in temporal dispersion and latency with location and stimulus ordinality.
} 
creases along the axon. Thus, failures will accumulate, causing $\Delta \mathrm{V}_{\mathrm{tr}}$ depression to intensify with distance from the stimulation electrode.

Using a high-speed CMOS camera developed recently by one of us (M.I.), with $100 \times 100$ pixels and frame rates of up to $10 \mathrm{kHz}$, we set out to determine the spatial pattern of $\Delta \mathrm{V}_{\mathrm{tr}}$ modulation to distinguish between these different mechanisms. Although morphological variations in pituitary size and shape preclude the averaging of results from multiple preparations, the image data discussed below represent the typical behavior seen with five different preparations. Figure $5 A$, panel 1 , shows the resting fluorescence of the preparation stained by bath application of JPW 1114 . The stimulation electrode is located slightly outside the recording area, as indicated by the bar. Figure $5 A$, panels $1-11$, are the false-color images of the change in resting fluorescence $(\Delta F)$ as the first population action potential sweeps from the top to the bottom across the preparation. As is evident from the sequence in Figure $5 A$, the first action potential spreads over the entire tissue.

The data permit us to obtain an approximate estimate for the propagation speed of the action potential under our recording conditions. The peak of the action potential traverses the $\sim 800 \mu \mathrm{m}$ distance from the top to the bottom of the preparation in $5 \mathrm{msec}$, corresponding to a conduction velocity of $160 \mathrm{~mm} / \mathrm{sec}$ at room temperature. This is approximately half the value determined from extracellular recordings of the compound action potential at $36^{\circ} \mathrm{C}$ (Nordmann and Stuenkel, 1986) and compares very well with the value of $190 \mathrm{~mm} / \mathrm{sec}$ obtained from numerical simulations of action potential propagation in this preparation (Jackson and Zhang, 1995).

The fluorescence difference images provide an optical record of the spatiotemporal pattern of action potential invasion. To discern changes in the spatial invasion of the action potential during repeated stimulation, we compared the frames representing the peak intensity of the spatially averaged action potential after the $2 \mathrm{nd}, 5 \mathrm{th}, 10 \mathrm{th}, 20 \mathrm{th}, 30 \mathrm{th}$, and 40 th stimuli. Figure $5 B$ shows this sequence of frames for 10,25 , and $40 \mathrm{~Hz}$ stimulus trains. All three sequences illustrate the overall decline in depolarization with ordinality (repeated stimulation), with the rate of decline increasing with stimulus frequency. This result confirms the behavior observed in the single photodiode recordings shown in Figure 3, $A$ and $C$. More importantly, within the limits of spatial resolution for these recordings $\left(8 \mu \mathrm{m}^{2} /\right.$ pixel $)$, the reduction in action potential amplitude appears spatially uniform. There is, however, some residual spatial heterogeneity in the optical records, with apparent hot spots lateral to the midline on both sides of the preparation (e.g., Fig. $5 B$, frame 1). These spatial variations in the $\Delta \mathrm{F}$ records of the first stimulus are not readily interpretable because they can arise from a combination of non- uniform dye staining and variations in the local density of excitable membrane area (axons and nerve terminals).

To confirm or disprove the visual impression of spatially uniform amplitude modulation, we analyzed quantitatively traces of local $\Delta \mathrm{V}_{\mathrm{tr}}$ changes. As indicated in Figure $6 \mathrm{~A}$, we extracted fluorescence versus time traces at three different locations on the preparation: close to the stimulation electrode (top), near the center of the preparation (center), and at the end farthest from the stimulation electrode (bottom). To improve the signal-tonoise ratio, the image data were locally averaged over an area of $10 \times 10$ pixels. As with the single photodiode data, we determined the $\Delta \mathrm{V}_{\mathrm{tr}}$ amplitude ratios of the $n$th to the first stimulus, $\Delta \mathrm{V}_{\mathrm{tr}, \mathrm{n}} / \Delta \mathrm{V}_{\mathrm{tr}, 1}$, for all three stimulation frequencies but now at each of the three locations independently (Fig. $6 \mathrm{~B}$ ). This analysis intrinsically accounts for variations in the local density of excitable membrane. Within the noise, the amplitude modulation of $\Delta \mathrm{V}_{\mathrm{tr}}$ transients is completely independent of location. This remains true for all three frequencies investigated here. A comparable analysis of local $\Delta \mathrm{V}_{\mathrm{tr}}$ modulation at the two apparent hot spots in Figure $5 B$ (left, right) yielded identical results (Fig. $6 B$ ). Therefore, the weak residual heterogeneity of the optical response 


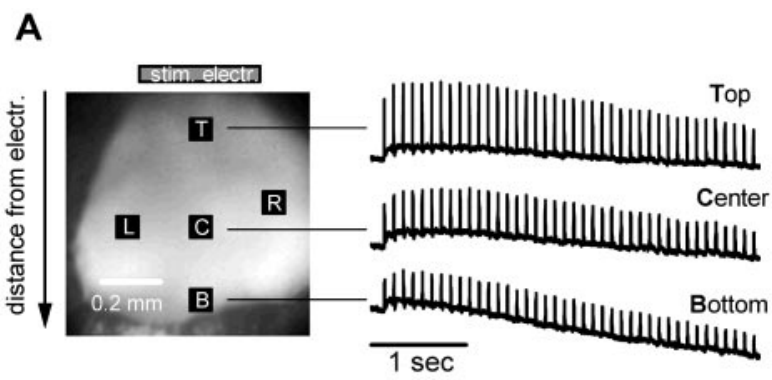

B

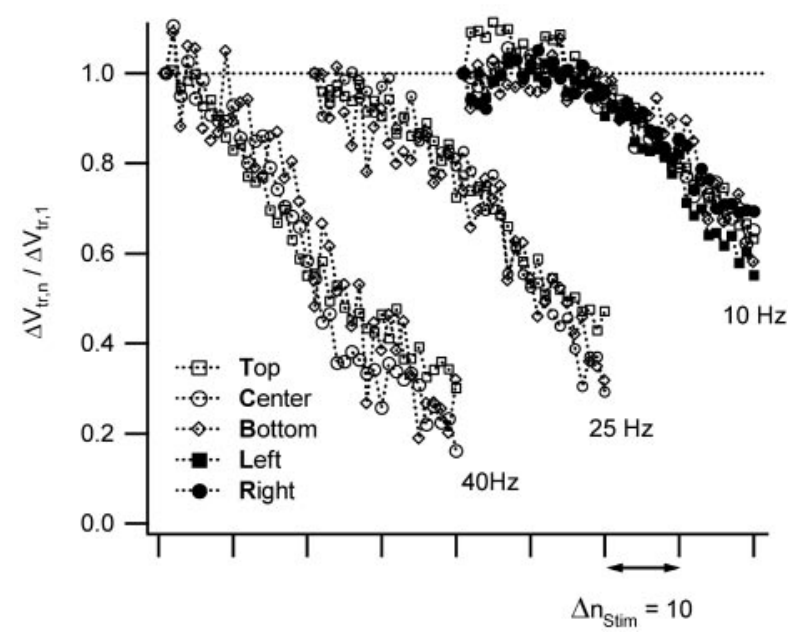

Figure 6. Local changes in tissue excitability as a function of stimulation ordinality and frequency. $A$, The three transmembrane voltage versus time traces shown to the right were obtained by locally averaging camera recordings over an area of $10 \times 10$ pixels. Size and location of each area [Top (T), Center (C), Bottom (B)] are indicated on the image of the preparation to the left. Two additional traces (Left, Right), representing apparent hot spots of response (compare with Fig. 5B, panel 1), were also extracted and analyzed. stim. electr., Stimulation electrode. $B$, Modulation of the voltage transient ratios, $\Delta \mathrm{V}_{\mathrm{tr}, \mathrm{n}} / \Delta \mathrm{V}_{\mathrm{tr}, 1}$, of the data in $A$. For clarity, the traces at 25 and $10 \mathrm{~Hz}$ are displaced from the origin by two and four $x$-axis ticks, respectively. Tick marks on the $x$-axis denote an increment in ordinality by 10 stimuli. The modulation of the $\Delta \mathrm{V}_{\operatorname{tr}}$ amplitudes is identical and independent of location, frequency, and ordinality. The local amplitude modulation at the hot spots ( $L, R$, shown here for $10 \mathrm{~Hz}$ only) is indistinguishable from that at the other loci.

seen in Figure 5, $A$ and $B$, arises from density variations of stained membrane, excitable membrane, or both.

\section{Changes in waveform of the population spike}

Besides the amplitude modulation within a train, the waveform of the population action potential also changes significantly with stimulus frequency, ordinality, and recording location. These changes fall into two distinct categories: altered temporal dispersion, which modifies the summation of individual action potentials into the population spike, and changes in the waveform of the underlying action potentials themselves.

For any given ordinality, the width of the $\Delta \mathrm{V}_{\mathrm{tr}}$ spike is much narrower proximal to the stimulation electrode (Fig. $7 A$, top) than at the two distal locations (Fig. 7A, center, bottom). Broadening at the distal locations is approximately symmetric around the peak of the population spike, increases further with stimulus ordinality, and is accompanied by a temporal shift (increased latency) of the peak position. These properties suggest that distal broadening originates from the temporal dispersion of the propagating excitation wave, traveling via different axonal pathways to the recording location. The increase in temporal dispersion of the locally recorded population spike with distance is already evident in the first response to a stimulus train (Fig. 7A, compare first spike, top to bottom). The morphological substrate for this initial rise in temporal dispersion is undoubtedly the intrinsic axonal branching pattern in the neurohypophysis.

The temporal dispersion of the population action potential is not constant in time, either; the latency and the width of the $\Delta \mathrm{V}_{\mathrm{tr}}$ peaks increase throughout any given stimulus train (Fig. 7A,B). The fixed axonal branching morphology cannot account for this dynamic component of temporal dispersion. Furthermore, the dynamic broadening at more distal locations is augmented by raising stimulation frequency (Fig. $7 B$ ). As a consequence of the frequency and ordinality dependence, the time to peak of the population spike can easily increase by several milliseconds at the more distal locations, reducing the speed of action potential propagation appreciably. The spatial variation in temporal dispersion of the population action potential is in stark contrast to its spatially homogeneous amplitude modulation.

The optical traces also suggest that there are changes in the waveform of individual action potentials. The optical transients, $\Delta \mathrm{V}_{\mathrm{tr}}$, at the most proximal location (Fig. $7 B$, top) have quite similar rise times but broaden noticeably at the falling flank of the action potential (Fig. 7A, top). As previously noted (Gainer et al., 1986), this behavior suggests broadening of the individual action potential waveform and is equivalent to the spike broadening observed in electrophysiological recordings from the neurohypophysis (Bourque, 1990; Jackson et al., 1991). Broadening of the individual action potential is masked at the more distal locations by the rapidly increasing temporal dispersion of the population spike.

\section{Calcium dependence of $\Delta V_{t r}$ amplitude modulation}

The spatially homogeneous modulation of $\Delta \mathrm{V}_{\mathrm{tr}}$ amplitudes is clearly inconsistent with failures of action potential propagation (Fig. $4 D$ ) but is compatible with several of the alternative models. One intriguing mechanism is the reported failure of nerve terminals and in-line varicosities to support active responses after repeated stimulation (Bourque, 1990; Bielefeldt and Jackson, 1993). This local loss of excitability is apparently related to a slowly activating, $\mathrm{Ca}^{2+}$-dependent $\mathrm{K}^{+}$conductance (Bielefeldt and Jackson, 1993). No selective toxin for this $\mathrm{K}^{+}$channel has been identified. However, interference with $\mathrm{Ca}^{2+}$ influx strongly affects the rate of these local excitability failures (Bourque, 1990; Bielefeldt and Jackson, 1993). Figure 8 quantitates the effects of the $\mathrm{Ca}^{2+}$ blocker $\mathrm{CdCl}_{2}$ and the L-type $\mathrm{Ca}^{2+}$ channel agonist $(S-)$ Bay-K 8644 on the macroscopically observed modulation of $\Delta \mathrm{V}_{\mathrm{tr}}$ amplitudes. Strikingly, $100 \mu \mathrm{M} \mathrm{CdCl}_{2}$ nearly completely abolishes both the facilitation and depression of $\Delta \mathrm{V}_{\mathrm{tr}}$ amplitudes. Only during $40 \mathrm{~Hz}$ stimulation does a residual component of depression persist (Fig. $8 C$ ). The $\mathrm{CdCl}_{2}$ result implies that $\mathrm{Ca}^{2+}$ influx is required for both the facilitation and depression of $\Delta \mathrm{V}_{\mathrm{tr}}$. Augmenting $\mathrm{Ca}^{2+}$ entry with Bay-K 8644 does not alter facilitation but, as expected (Bielefeldt and Jackson, 1993), enhances depression of $\Delta \mathrm{V}_{\mathrm{tr}}$ amplitudes, particularly at intermediate frequencies. The depicted sensitivity of $\Delta \mathrm{V}_{\mathrm{tr}}$ amplitudes to both $\mathrm{CdCl}_{2}$ and Bay-K 8644 supports the idea that the local loss of active responses in nerve terminals and varicosities, mediated by a Ca-activated $\mathrm{K}$ conductance, generates the macroscopic changes in the population action potential seen in our optical data. 
A
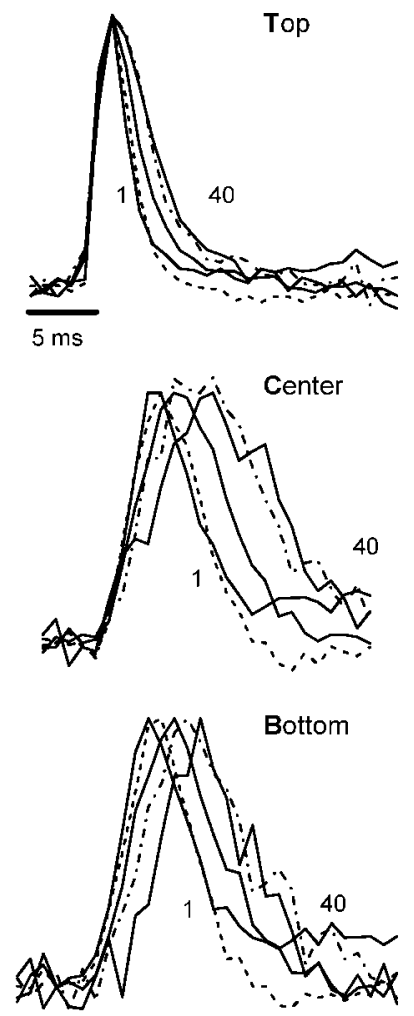

B

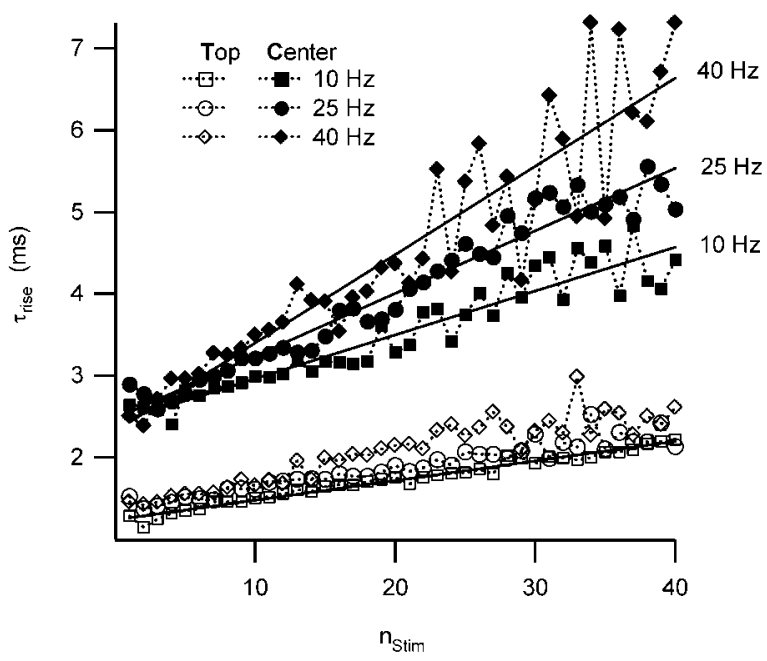

Figure 7. Changes in action potential waveform: action potential broadening versus temporal dispersion of the population action potential. A, Superposition of the waveform of the 1st (solid line), 10th (dotted line), 20th (solid line), 30th (dashed, dotted line), and 40th (solid line) transients of locally extracted traces from a $25 \mathrm{~Hz}$ stimulus train. The amplitudes of the transients have been matched to one another. The asymmetric broadening of the population action potential at the top is indicative of a small broadening of the individual action potentials, which develops gradually with ordinality. In contrast, the increase in peak latency and symmetric broadening of the peak at the more distal locations is indicative of temporal dispersion of the transients. With increasing distance from the stimulation electrode, temporal dispersion rapidly comes to dominate the changes in the population spike waveform. $B$, Temporal dispersion versus location. Changes in the rise time $\left(\tau_{\text {rise }}\right)$ of the local $\Delta V_{\text {tr }}$ transients in $A$ at a location near (open symbols) and far (filled symbols) from the recording electrode are shown. The difference in rise time for $n_{\text {stim }}=1$ indicates the intrinsic difference in temporal dispersion attributable to the axonal branching morphology (see Results). The dynamicincrease of rise times with $n_{\text {stim }}$ is approximately constant at the proximal location (overlapping open traces) but accelerates noticeably with frequency at the more distant location. Rise times were obtained from Gaussian fits to the rising phase and peak region of individual optical transients. Straight lines represent least-squares fits through the rise time data.

\section{Discussion}

$\Delta \mathrm{V}_{\mathrm{tr}}$ and $\Delta\left[\mathrm{Ca}^{2+}\right]_{\mathrm{tr}}$ amplitude modulation and the role of action potential broadening

The results of our optical experiments indicate that both the amplitude and the waveform of the population action potential are noticeably altered during repetitive stimulation. Particularly surprising is the strong amplitude modulation. During repeated stimulation at low frequencies, some facilitation emerges. The dominant effect, however, is amplitude depression, which develops after persistent stimulation even at low frequencies and accelerates dramatically with increasing stimulation frequency (see Fig. $3 C$ ). The combined paired pulse recordings of $\Delta \mathrm{V}$ and $\Delta \mathrm{Ca}^{2+}$ transients reveal that $\Delta \mathrm{V}_{\mathrm{tr}}$ depression is bound to contribute to $\Delta \mathrm{Ca}_{\mathrm{tr}}$ depression. At the same time, the lack of $\Delta \mathrm{V}_{\mathrm{tr}}$ facilitation and onset of $\Delta \mathrm{V}_{\mathrm{tr}}$ depression during $\Delta \mathrm{Ca}_{\mathrm{tr}}$ facilitation (see Fig. $2 D$, regions III, II, respectively) indicate that $\Delta \mathrm{V}_{\mathrm{tr}}$ modulation cannot account for all aspects of $\Delta \mathrm{Ca}_{\mathrm{tr}}$ variation. Other mechanisms involved in $\Delta\left[\mathrm{Ca}^{2+}\right]_{\text {tr }}$ depression might include calcium channel inactivation (Branchaw et al., 1997) or buildup of extracellular potassium (Leng and Shibuki, 1987).

In addition to the amplitude modulation, the optical data reveal significant changes in the waveform of the population action potential. The activity- and site-dependent rise in temporal dispersion dominates these waveform changes. Dispersion and increasing latency are robust features of action potential invasion in the neurohypophysis, observed consistently in our optical experiments, during electrophysiological recordings of the compound action potential (Nordmann and Stuenkel, 1986), and in action potentials recorded from individual nerve terminals (Bourque, 1990). Any model of the amplitude modulation of the population action potential also has to account for this dynamic increase in latency.

Could action-potential broadening give rise to the large facilitation of $\Delta \mathrm{Ca}^{2+}$ transients? Increased calcium influx caused by action potential broadening was one of the earliest mechanisms invoked to explain the frequency dependence of hormone release (Gainer et al., 1986; Bourque, 1990; Jackson et al., 1991). It has recently received renewed attention at hippocampal synapses (Geiger and Jonas, 2000). We have previously argued that the rapid onset of $\Delta \mathrm{Ca}^{2+}$ facilitation with ordinality does not agree well with the gradual development of action potential broadening in the neurohypophysis seen in optical and electrophysiological recordings (Gainer et al., 1986; Bourque, 1990; Jackson et al., 1991). Consistent with our reservations, broadening of the optically recorded $\Delta \mathrm{V}_{\mathrm{tr}}$ waveform is most noticeable toward the end of stimulation trains and at high frequencies (Fig. 7A, top), similar to the results from electrophysiological measurements of broadening (Bourque, 1990, his Fig. 6). High stimulation frequencies and stimulus ordinality, however, are both conditions associated with large depression, not facilitation, of $\Delta \mathrm{Ca}^{2+}$ transients (Muschol and Salzberg, 2000, their Fig. 3).

\section{Mechanisms of $\Delta \mathrm{V}_{\mathrm{tr}}$ modulation}

It was our a priori expectation that the dramatic decline in $\Delta \mathrm{V}_{\text {tr }}$ amplitudes (up to 75\%; see Fig. 3C) reflected failures and block of action potential propagation at axonal varicosities or branch points (Fig. 4, model $D$ ). This expectation was based on three previous observations. First, invasion can be altered significantly in the amphibian neurohypophysis (Obaid and Salzberg, 1996). Second, during action potential propagation in the rat neurohypophysis, sharp electrode recordings from nerve terminals revealed a sudden transition from active responses to catastrophic failure (Bourque, 1990, his Fig. 9). Third, local current injection 
A

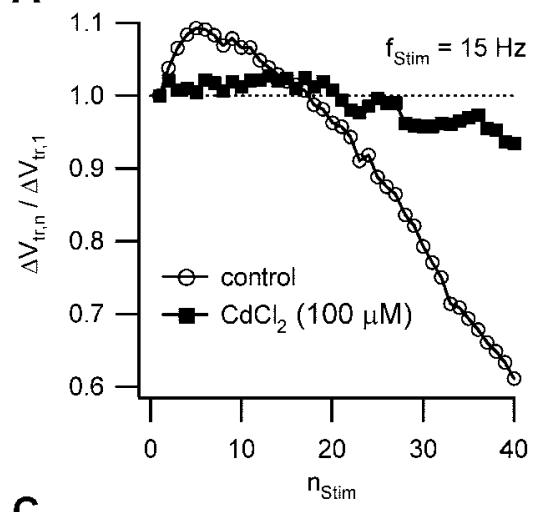

C

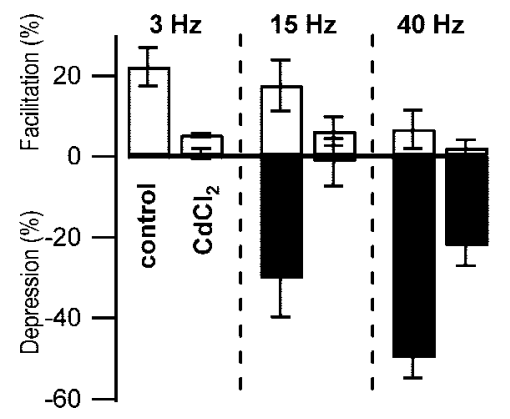

B

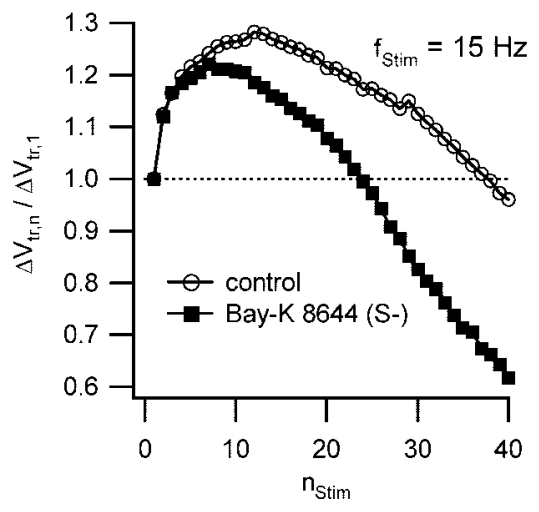

D

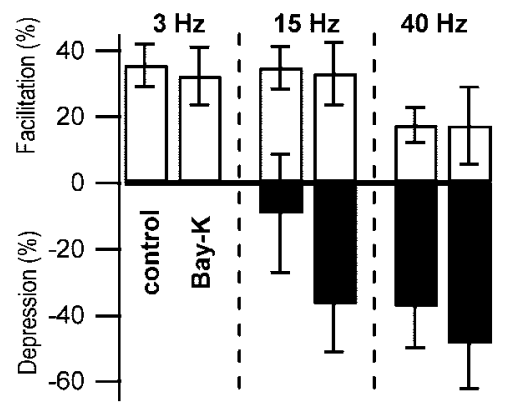

Figure 8. Calcium dependence of $\Delta \mathrm{V}_{\mathrm{tr}}$ modulation. Modulation of $\Delta \mathrm{V}_{\mathrm{tr}}$ amplitude ratios, $\Delta \mathrm{V}_{\mathrm{tr}, \mathrm{n}} / \Delta \mathrm{V}_{\mathrm{tr}, 1}$, recorded from a single preparation before (open circles) and after (filled squares) application of $\mathrm{CdCl}_{2}(100 \mu \mathrm{m} ; A)$ or the L-type $\mathrm{Ca}^{2+}$ agonist $(S-)$ Bay-K $8644(10 \mu \mathrm{m} ; B)$ is shown. The amplitudes of the individual transients used to generate these data were extracted from optical recordings of voltage changes as shown in Figure $3 A$. To quantify the effect of the drugs, we compared their impact on the maximum of the facilitation and the depression of $\Delta \mathrm{V}_{\mathrm{tr}}$ amplitudes during a continuous train of 40 stimuli at three different frequencies. Each pair of bars in the charts below summarizes the average values ( $n=4$; error bar indicates 1 SD) of maximal facilitation (upward, open bars) and depression (downward, filled bars) in control or after exposure to either $\mathrm{CdCl}_{2}(100 \mu \mathrm{m} ; \mathrm{C})$ or $(S-)$ Bay-K $8644(10 \mu \mathrm{m} ; D)$. The effects of the drugs are shown separately for each of the three indicated frequencies.

into nerve terminals frequently failed to evoke active responses during repeated stimulation (Bielefeldt and Jackson, 1993). Block of propagation, however, should preferentially decrease the amplitude of the population action potential at locations distal to the stimulation electrode (Fig. 4, model D). Clearly, this does not occur; instead, the amplitude of the population action potential decreased uniformly throughout the neurohypophysis (see Figs. $5,6)$.

In principle, failures of action potential initiation at the site of simulation (Fig. 4, model $C$ ), combined with enhanced refractoriness to account for the increase in latency (Fig. 4, model A), could produce the pronounced amplitude depression of the population action potential. Two observations argue against this interpretation in the neurohypophysis. Most significantly, Bourque (1990) noted that, although local loss of excitability was readily observed in nerve terminals and varicosities, action potentials recorded from the axons of the infundibular stalk showed no signs of refractoriness or propagation failure. In addition, the increased latency with stimulus ordinality, far from the stimulation electrode (Fig. 7A,B), seems to imply that distal locations would be more prone to remain below the threshold for action potential generation. Therefore, failures should arise there preferentially and not at the stimulation site. This is at variance with the spatially uniform decline in the population action potential seen in our optical data (Fig. $6 B$ ).

The effects of $\mathrm{CdCl}_{2}$ and Bay-K 8644 on $\Delta \mathrm{V}_{\mathrm{tr}}$ modulation (Fig. 8 ), conversely, argue for a significant role for local excitability failures in the macroscopically observed modulation of $\Delta \mathrm{V}_{\mathrm{tr}}$ amplitudes. Given the limited resolution of our optical recordings $\left(8 \mu \mathrm{m}^{2}\right)$ and the uniform distribution of axons, nerve terminals, and varicosities within the neurohypophysis, local loss of excitability is consistent with the spatially homogeneous change in the amplitude of the population action potential. The hypothesis of selective excitability loss in terminals and varicosities is further buttressed by evidence that neurohypophysial nerve terminals have populations of $\mathrm{K}^{+}$ and $\mathrm{Ca}^{2+}$ channels that are electrophysiologically and pharmacologically different from those in their magnocellular somata (Fisher and Bourque, 1995; Dopico et al., 1999). The distinctive populations of $\mathrm{Ca}^{2+}$ and $\mathrm{K}^{+}$channels in terminals and varicosities would then provide the macromolecular machinery underlying local loss of excitability. The restriction of local excitability failures to axonal end points (Fig. 4, model B), however, is inconsistent with morphological studies that indicate that a substantial fraction of the secretory sites are in-line varicosities (Jackson, 1993), which do inactivate (Bourque, 1990; Bielefeldt and Jackson, 1993). Therefore, the action potential has to propagate through passive varicosities without failing.

These considerations suggest to us the modified version of "local loss of excitability" shown schematically in Figure $4 E$. Consistent with existing electrophysiological evidence (Bourque, 1990; Bielefeldt and Jackson, 1993), we assume that repeated stimulation eventually fails to evoke active responses in some in-line varicosities and nerve terminals. The combined cable properties of axonal segments and varicosities, however, permit the action potential to spread passively through varicosities and, yet, to evoke an actively propagated response after emerging distal to the swellings. The latter conjecture is supported by numerical simulations by Jackson and Zhang (1995), which indicate that action potentials can propagate through in-line varicosities in the neurohypophysis, even when the active response in the varicosity itself is suppressed by inhibitory input. We have termed this model "stuttering conduction" because it also provides a straightforward explanation for the dynamic retardation of the action potentials. The active propagation of the action potential is progressively retarded by the increasing number of passive structures that the voltage signal has to negotiate. This activity-dependent rise in local latency should increase dispersion with distance from the stimulating electrode, consistent with our optical data (Fig. 7A). Latency will also tend to increase during sustained stimulation, as more and more secretory varicosities become inactivated. (Nordmann and Stuenkel, 1986; Bourque, 1990; this study, Fig. 7B). In short, stuttering conduction provides a compelling, selfconsistent explanation for all the experimental observations on action potential propagation and local loss of excitability in the neurohypophysis. 


\section{Conclusions}

Sustained stimulation of the mammalian neurohypophysis induces a frequency-dependent modulation of the population action potential. The magnitude of $\Delta \mathrm{V}_{\mathrm{tr}}$ depression, in particular, establishes the loss of excitable membrane area as the dominant contribution to the modulation. The onset and magnitude of the depression in excitability is closely related to concurrent changes in intraterminal calcium transients. Excitability changes, therefore, play a significant role in the short-term depression of peptide release from the mammalian neurohypophysis during physiologically relevant episodes of stimulation. The spatial pattern of excitability depression reveals that the amplitude of the population action potential decays uniformly everywhere, whereas temporal dispersion increases with both the distance from the stimulation site and the stimulus ordinality. To account for these two specific and robust features of the optical data and the existing electrophysiological evidence we have proposed a model of stuttering conduction.

It remains to be seen whether local excitability changes as a mechanism of short-term plasticity are limited to the neurohypophysis or to peptide release. For example, the observed retardation of the action potential could be considered a peculiarity of the specialized morphology of secretory varicosities arrayed linearly along the axons. This morphology is, however, strongly reminiscent of the en passant synapses between parallel fibers and Purkinje neurons in the cerebellum (Xu-Friedman et al., 2001) and could alter the spatial and temporal summation of synaptic inputs from cerebellar granule cells. Finally, the calciumdependent regulation of the local excitability of nerve terminals and secretory swellings suggests yet another role for calcium in neuropeptide release. The combination of $\mathrm{Ca}^{2+}$ channels and $\mathrm{Ca}^{2+}$-dependent $\mathrm{K}^{+}$channels implicated in these local excitability changes (Bourque, 1990; Bielefeldt and Jackson, 1993), however, is commonly found in cells with $\mathrm{Ca}^{2+}$-triggered exocytosis (Jackson, 1995). Local regulation of presynaptic excitability, thus, could emerge as a natural and possibly widespread mechanism for evoking short-term plasticity in other areas of the CNS.

\section{References}

Antic S, Zecevic D (1995) Optical signals from neurons with internally applied voltage-sensitive dyes. J Neurosci 15:1392-1405.

Antic S, Loew L, Wuskell J, Zecevic D (1992) Voltage-sensitive dyes for intracellular application. Biol Bull 183:350-351.

Antic S, Major G, Zecevic D (1999) Fast optical recordings of membrane potential changes from dendrites of pyramidal neurons. J Neurophysiol 82:1615-1621.

Betz WJ, Bewick GS (1992) Optical analysis of synaptic vesicle recycling at the frog neuromuscular junction. Science 255:200-203.

Bicknell RJ (1988) Optimizing release from peptide hormone secretory nerve terminals. J Exp Biol 139:51-65.

Bielefeldt K, Jackson MB (1993) A calcium-activated potassium channel causes frequency-dependent action potential failures in a mammalian nerve terminal. J Neurophysiol 70:284-298.

Bourque CW (1990) Intraterminal recordings from the rat neurohypophysis in vitro. J Physiol (Lond) 421:247-262.

Branchaw JL, Banks MI, Jackson MB (1997) $\mathrm{Ca}^{2+}$ - and voltage-dependent inactivation of $\mathrm{Ca}^{2+}$ channels in nerve terminals of the neurohypophysis. J Neurosci 17:5772-5781.

Cazalis M, Dayanithi G, Nordmann JJ (1985) The role of patterned burst and interburst interval on the excitation-coupling mechanism in the isolated rat neural lobe. J Physiol (Lond) 369:45-60.

Cohen LB, Salzberg BM (1978) Optical measurements of membrane potential. Rev Physiol Biochem Pharmacol 83:35-88.
Davila HV, Cohen LB, Salzberg BM, Shirvastav BB (1974) Changes in ANS and TNS fluorescence in giant axons from Loligo. J Membr Biol 15:28-46.

Dopico AM, Widmer H, Wang G, Lemos JR, Treistman SN (1999) Rat supraoptic magnocellular neurones show distinct large conductance, $\mathrm{Ca}^{2+}$. activated $\mathrm{K}^{+}$channel subtypes in cell bodies versus nerve endings. J Physiol (Lond) 519:101-114.

Fisher TE, Bourque CW (1995) Distinct $\omega$-agatoxin-sensitive calcium currents in somata and axon terminals of rat supraoptic neurones. J Physiol (Lond) 489:383-388.

Gainer H, Wolfe Jr SA, Obaid AL, Salzberg BM (1986) Action potentials and frequency-dependent secretion in the mouse neurohypophysis. Neuroendocrinology 43:557-563.

Geiger JRP, Jonas P (2000) Dynamic control of presynaptic $\mathrm{Ca}^{2+}$ inflow by fast-inactivating $\mathrm{K}^{+}$channels in hippocampal mossy fiber boutons. Neuron 28:927-939.

Jackson MB (1993) Passive current flow and morphology in the terminal arborizations of the posterior pituitary. J Neurophysiol 69:692-702.

Jackson MB (1995) Presynaptic excitability. Int Rev Neurobiol 38:201-251.

Jackson MB, Zhang SJ (1995) Action potential propagation and propagation block by GABA in rat posterior pituitary nerve terminals. J Physiol (Lond) 483:597-611.

Jackson MB, Konnerth A, Augustine GJ (1991) Action potential broadening and frequency-dependent facilitation of calcium signals in pituitary nerve terminals. Proc Natl Acad Sci USA 88:380-384.

Konnerth A, Obaid AL, Salzberg BM (1987) Optical recording of electrical activity from parallel fibres and other cell types in skate cerebellar slices in vitro. J Physiol (Lond) 393:681-702.

Lattanzio Jr FA, Bartschat DK (1991) The effect of pH on rate constants, ion selectivity and thermodynamic properties of fluorescent calcium and magnesium indicators. Biochem Biophys Res Commun 177:184-191.

Leng G, Shibuki K (1987) Extracellular potassium changes in the rat neurohypophysis during activation of the magnocellular neurosecretory system. J Physiol (Lond) 392:97-111.

Muschol M, Salzberg BM (2000) Dependence of transient and residual calcium dynamics on action potential patterning during neuropeptide secretion. J Neurosci 20:6773-6780.

Nordmann JJ (1977) Ultrastructural morphometry of the rat neurohypophysis. J Anat 123:213-218.

Nordmann JJ, Stuenkel EL (1986) Electrical properties of axons and neurohypophysial nerve terminals and their relationship to secretion in the rat. J Physiol (Lond) 380:521-539.

Obaid AL, Salzberg BM (1996) Micromolar 4-aminopyridine enhances invasion of a vertebrate neurosecretory terminal arborization: optical recording of action-potential propagation using an ultrafast photodiodeMOSFET camera and photodiode array. J Gen Physiol 107:353-368.

Obaid AL, Koyano T, Lindstrom J, Sakai T, Salzberg BM (1999) Spatiotemporal patterns of activity in an intact mammalian network with single cell resolution: optical studies of nicotinic activity in an enteric plexus. J Neurosci 19:3073-3093.

Poulain DA, Wakerley JB (1982) Electrophysiology of hypothalamic magnocellular neurons secreting oxytocin and vasopressin. Neuroscience 7:773-808.

Rohr S, Salzberg BM (1994) Multiple site optical recordings of transmembrane voltage (MSORTV) in patterned growth heart cell cultures: assessing electrical behavior, with microsecond resolution, on a cellular and subcellular scale. Biophys J 67:1301-1315.

Salzberg BM (1983) Optical recording of electrical activity in neurons using molecular probes. In: Current methods in cellular neurobiology (Barker J, McKelvy J, eds), pp 139-187. New York: Wiley.

Salzberg BM, Obaid AL, Senseman DM, Gainer H (1983) Optical recording of action potentials from vertebrate nerve terminals using potentiometric probes provides evidence of sodium and calcium components. Nature 306:36-40.

Stuenkel EL (1994) Regulation of intracellular calcium and calcium buffering properties of rat isolated neurohypophysial nerve endings. J Physiol (Lond) 481:251-271.

Xu-Friedman MA, Harris KM, Regehr WG (2001) Three-dimensional comparison of ultrastructural characteristics at depressing and facilitating synapses onto cerebellar Purkinje cells. J Neurosci 21:6666-6672. 